\title{
Bacteriological Quality of Citrus Fruits (Morocco)
}

\author{
Khaled Attrassi
}

Research Laboratory in Education, Environment \& Health (ĖES), Regional Center of Education and Training Trades Rabat-Salé-Kenitra (CRMEF), 245, Kénitra, Morocco.

\begin{abstract}
In our research, we studied the bacteriological quality of citrus fruits. Thus, the bacterial microflora associated with citrus fruit is quite varied and diverse. It is usually a saprophyte flora easily be destroyed by moist heat. This plant belongs to the family Enterobacteriaceae, Vibrionaceae, Pseudomonadaceae, Micrococcaceae and Bacilaceae. This contamination is often untreated manure, organic fertilizer from a bad composting, irrigation water not clean. The hands of workers who come into contact with fruit are also a potential source of contamination across the production system of the juice, ie at all these stages: growing, harvesting, processing, packaging and transportation. This contamination is usually faecal origin toilet (hands of workers contaminated by fecal matter).
\end{abstract}

The presence of E. Coli and Streptococcus feacalis is evidence of such contamination. Indeed, the presence of these bacteria indicates that other pathogens present in the feces of sick individuals or healthy carriers may be present (Salmonella, Vibrio, Clostridia...) what constitutes a risk of contamination of the entire production. Among the most dangerous pathogens that can be seen quite often on the fruit, quoting E. coli O157: H7, Salmonella spp.... Colereae Vibrio, which are Gram negative, the wall, composed of lipopolysaccharide (LPS), is an endotoxin. The ingestion of endotoxin causes a water leak at the level of the enterocytes (cells of the intestine) and consequently dehydration of the individual addict.

Keywords - citrus fruits, contamination, microbiological quality, bacterial microflora. .

\section{Qualité bactériologique des fruits d'agrumes (Maroc)}

Résumé-Dans notre travail de recherche, nous avons étudie la qualité bactériologique des fruits d'agrumes. Ainsi, La microflore bactérienne associée aux fruits d'agrume est assez variée et diversifiée. Il s'agit généralement d'une flore saprophyte facilement destructible par la chaleur humide.

Cette flore appartient aux familles des Enterobactériaceae, Vibrionaceae, Pseudomonadaceae, Bacilaceae et Micrococcaceae. Cette contamination provient souvent du fumier non traité, d'engrais organique provenant d'un mauvais compostage, d'eau d'irrigation non propre. Les mains des ouvriers qui entrent en contact avec les fruits représentent aussi une source potentielle de contamination à la grandeur du système de production du jus, c'est à dire à toute ces étapes : culture, récolte, transformation, emballage et transport.

Cette contamination est généralement fécale originaire des toilettes (mains des ouvriers souillées par la matière fécale). La présence d'E.coli et de Streptococcus feacalis constitue une preuve de cette contamination. En effet, la présence de ces germes signifie que d'autres germes pathogènes présents dans la matière fécale des individus malades ou porteurs sains peuvent être présents (salmonelles, vibrions, clostridies....) ce qui constitue un risque de contamination de toute la production.

Parmi les germes pathogènes les plus redoutables qu'on peut rencontrer assez souvent sur les fruits, on cite E.coli O157: H7, Salmonella spp., Vibrio colereae..., qui sont des bactéries Gram négatif dont la paroi, formée des lipopolysaccharides (LPS), constitue une endotoxine. L'ingestion de ces endotoxines provoque une 
fuite d'eau au niveau des enterocytes (cellules de l'intestin) et par voie de conséquence une déshydratation de l'individu intoxiqué.

Mots clés—Fruits d'agrumes, contamination, qualité microbiologique, microflore bactérienne.

\section{INTRODUCTION}

Les infections des fruits d'agrumes par l'agent pathogène peuvent se produire aussi bien avant qu'après la récolte. Les infections des fruits d'agrumes sont initiées par des contaminations au champ, quelques jours à plusieurs semaines avant la récolte. Ces infections sont favorisées, entre autres, par un taux d'inoculation élevé dans l'air et par une grande humidité. La détérioration peut être limitée par des conditions défavorables de l'environnement et également par une résistance de l'écorce du fruit (Douyle et Padhye, 1989 ; Doyle et Cliver, 1990 ; Beuchat et Ryu, 1997 ; Jackson, 2001 ; Forrest, 2001).

Les portes d'entrées des agents pathogènes en cours et après la récolte (stockage) des agrumes sont les blessures et les microlésions accidentelles (Faber, 1989. Tabaght, 1994).

Dans cette partie de notre travail, nous allons examiner la qualité des fruits d'orange tout en exposant les principaux paramètres physico-chimiques, nutritionnels et techniques susceptibles d'influer sur la qualité. Nous allons mettre en relief l'importance de certaines espèces de bactéries dans la dégradation de la qualité des fruits d'orange.

\section{MATERIEL ET METHODES}

\section{1/ Matériel biologique}

\section{a/ Fruits des agrumes}

Les fruits, sujets de notre étude proviennent des plantes fruitières appartenant aux espèces suivantes :

* Clémentinier (Citrus clementina Hort. ex. Tan.),

* Mandarinier (Citrus reticulata Blanco),

* Oranger (Citrus sinensis Osb),

* Citronnier (Citrus limon L.),

* Pomelo (Citrus paradisi Macf.).

\section{2/ Echantillonnage}

* Fruits d'agrumes : pour le contrôle des agrumes nous avons choisi les niveaux suivants:

- station réception d'agrumes

- station brossage-lavage à l'avant et après sortie.

On prend $1 \mathrm{~kg}$ de fruit comme un échantillon représentatif. Les fruits sont lavés par l'eau distillée stérile à raison de $250 \mathrm{ml}$ pour $1 \mathrm{~kg}$ d'échantillon.
L'analyse de l'eau de lavage nous donne des indications sur le degré de contamination des fruits par des souches pathogènes (bactéries ou champignons).

3/ Culture et identification des microorganismes (bactéries et champignons)

\section{3-1/ Milieux de culture}

\section{3-1-1/ Milieu Orange Serum Agar (OSA)}

C'est un milieu de culture qui permet le développement de toute la flore qui exige des facteurs de croissance présents dans l'extrait d'orange.

Les bactéries, champignons, (levures et moisissures) adaptés aux jus d'orange se cultivent sur ce milieu.

Le milieu OSA contient en g/l (Tryptone, 10 ; extrait de levure, 3 ; extrait Orange, 5 ; Glucose, 4 ; Phosphate dipotassique, 3 ; Agar bactériologique, 17)

Le milieu est stérilisé à l'autoclave à $120^{\circ} \mathrm{C} / 15 \mathrm{~min}$, puis coulé en boîtes de Petri stérilisées à l'autoclave $\left(120^{\circ} \mathrm{C} / 30\right.$ min) ou à sec au four $\left(170^{\circ} \mathrm{C} / 1 \mathrm{~h}\right)$.

\section{3-1-2/ Gélose nutritive}

La gélose nutritive est un milieu qui convient à la culture des germes ne présentant pas d'exigences particulières. Ce milieu est composé de (en g/l ): (Peptone, 5 ; Extrait de viande, 1 ; Extrait de levure, 2 ; Chlorure de sodium, 5 ; Agar, 15)

Ce milieu est stérilisé à l'autoclave $\left(120^{\circ} \mathrm{C} / 15 \mathrm{~min}\right)$ et coulé en boîte de Petri ou en tube incliné pour la conservation des souches pures. Dans ce cas, la conservation se fait à $4^{\circ} \mathrm{C} / 1$ mois.

\section{3-1-3/ Milieu à base de pomme de terre (PSA)}

Des petits cubes de $200 \mathrm{~g}$ de pommes de terre non pelées et vieilles de préférences, sont lavés, coupés et placés dans un litre d'eau portée à ébullition pendant 1 heure. Après ébullition, les pommes de terre sont écrasées puis filtrées et le volume est complété à 1 litre.

Ce mélange est ensuite additionné de $15 \mathrm{~g}$ de saccharose et $20 \mathrm{~g}$ d'agar. Le $\mathrm{pH}$ du milieu est ajusté à 5,6 et stérilisé à l'autoclave à $120^{\circ} \mathrm{C} / 15 \mathrm{~min}$.

Le milieu PSA est utilisé pour l'isolement, la culture et le dénombrement des champignons. 
Ce milieu est particulièrement recommandé pour la mise en évidence des contaminations dans les produits alimentaires (ex: jus d'orange).

\section{3-1-4/ Bouillon nutritif}

Ce milieu a la même composition que la gélose nutritive mais sans agar bactériologique.

Ce milieu, coulé en tubes et stérilisé à l'autoclave, sert généralement aux préparations de précultures et aux enrichissements.

\section{3-1-5/ Milieu TCBS}

C'est un milieu sélectif qui sert à isoler les germes de vibrions après enrichissement sur milieu riche contenant 40 $\mathrm{g}$ de peptone et $60 \mathrm{~g}$ de $\mathrm{Nacl}$ par litre.

Le milieu TCBS a la composition suivante (en g/l ) : (Peptone, 10 ; Extrait de levure, 5 ; Citrate de sodium, 10 ; Thiosulfate de sodium, 10 ; Chlorure de sodium, 10 ; Bile de bœuf, 8 ; Citrate ferrique, 1 ; Saccharose, 20 ; Bleu de thymol, 0,04 ; Bleu de bromothymol, 0,04 ; Agar ; 14). Ce milieu est chauffé jusqu'à ébullition mais non autoclavé.

Après ensemencement, les boîtes sont incubées 15 à 18 heures à $35^{\circ} \mathrm{C}$, période suffisante pour l'apparition des colonies des vibrions alors que la totalité des autres germes est inhibée durant les premières heures d'incubation. Les colonies de vibrions sont caractéristiques de quelques espèces:

- V. parahaemolyticus : petite colonie à centre vert ou bleu à bord incolore

- V. cholerea : petite colonie jaune

- V. aglinolyticus : grosse colonie jaune-brun

Les colonies suspects sont repiquées sur milieu Kligler salé à $3 \%$ pour identification (voir milieux d'identification).

\section{3-1-6/ Milieu King A et King B.}

Ces milieux servent à l'isolement des $P$ seudomonas.

Les milieux King A (ou P) et King B (ou F) permettent de différencier les espèces pigmentées de Pseudomonas.

Ces milieux ont la composition suivante (en $\mathrm{g} / \mathrm{l}$ ): pour milieu King A (ou P) on a: (Peptone de gélatine, 20 ; Glycérol, 10 ; Sulfate de potassium, 10 ; Chlorure de magnésium anhydre, 1,4;Agar, 15). La stérilisation se fait à l'autoclave à $120^{\circ} \mathrm{C} / 15 \mathrm{mn}$.

Pour le milieu de King B (ou F) on a : (Protéose peptone $\mathrm{n}^{\circ} 3$ ou polypeptone (BBL), 20 ; Glycérol, 10 ; Phosphate, 1,5 ; Sulfate de magnésium $\left(7 \mathrm{H}_{2} \mathrm{O}\right), 1,5 ;$ Agar, 15). Ce milieu est stérilisé à l'autoclave à $120^{\circ} \mathrm{C} / 15 \mathrm{~min}$.

Le milieu A favorise la production de pyocyanique du bacille pyocyanique ou Ps. aerogenosa alors que le milieu
B favorise la fluorescine (Pyoverdine) de Ps. fluorescens, Ps.putida, et divers autres Pseudomonas.

Une boîte de milieu A et une boîte de milieu B sont ensemencées avec une anse de culture prise dans un bouillon nutritif. Après incubation à $30^{\circ} \mathrm{C} / 4$ jours, les colonies suspectes sont repiquées sur milieu Kligler pour la suite d'identification.

\section{3-2/ Ensemencement}

\section{3-2-1/ Ensemencement pour isoler des souches}

Pour isoler une souche, en pratique un ensemencement par épuisement sur un milieu de culture approprié à partir des fruits, de jus ou autres.

Après incubation à $30^{\circ} \mathrm{C} / 48 \mathrm{~h}$, les colonies isolées sont ensemencées à nouveau comme précédemment pour s'assurer de la pureté de la souche.

Les souches diffèrent normalement par leurs aspects culturaux (forme de la colonie, aspect, couleur, élévation, consistance,...). Chaque type de colonie est repiqué séparément et conservé sur gélose nutritive inclinée (pour les bactéries) ou sur PSA incliné (pour les levures et les moisissures) en vue d'une identification complète.

\section{3-2-2/ Dénombrement de la microflore du jus et des fruits.}

Une série de dilution du jus semi-fini ou de l'eau de lavage est réalisée pour obtenir une suspension bactérienne dénombrable sur milieu solide en boîte de Petri. Des dilutions de 10 en 10 sont réalisées et l'ensemencement se fait par étalement de $0,1 \mathrm{ml}$ de chaque dilution sur le milieu de culture OSA dans des conditions aseptiques.

Les boîtes sont ensuite incubées inversées dans l'étuve à 30 ${ }^{\circ} \mathrm{C} / 48 \mathrm{~h}$.

L'ensemencement peut être aussi réalisé par la méthode d'ensemencement en masse, mais cette méthode sous estime la taille de la population par le fait que les souches très exigeantes en oxygène ne se développent pas.

Chaque souche vivante introduite dans la masse d'un milieu gélosé favorable donne en principe naissance à une colonie repérable à l'œil nu. En conséquence, si un produit ou sa dilution est ensemencé dans ce milieu de culture, le nombre de colonies développées correspond au nombre de micro-organismes présent dans le volume inoculé.

\section{3-3/ Identification}

\section{3-3-1// Etude macro et microscopique}

\section{a/ Observation des colonies}

Après incubation, tous les caractères macroscopiques des colonies sont notés.

- Forme de la colonie : circulaire, irrégulière ou rhizoïde. 
- Aspect : punctiforme (<1 mm de diamètre), moyenne, grande ou envahissante.

- Opacité : transparence, translucidité.

- Elévation : colonie plate, convexe, centrée, surélevée.

- Surface : lisse, rugueuse, émoussée, brillante, sèche, poudreuse, plissée, crémeuse.

- Bord : entier, ondulé, lobé, denté, rhizoïde, crénelé.

- Consistance : visqueuse ou granulaire.

- Odeur : présence ou absence d'odeur caractéristique.

Chaque type de colonie est repiqué stérilement dans un tube de bouillon nutritif stérile, après purification.

\section{b/ Observation microscopique.}

* A l'état frais: chaque souche (bactéries) est observée au microscope entre lame et lamelle avec ou sans colorants (bleu de méthylène). On note généralement la forme, la taille, l'arrangement et pour les champignons on note en plus la structure.

* Après coloration de Gram pour les bactéries: La coloration de Gram permet de séparer deux grands groupes de bactéries: Gram + et Gram -. Les premiers ont une paroi épaisse et sans LPS, ces types de bactéries se colorent en violet car l'alcool ne pénètre pas dans la cellule alors que les seconds ayant une paroi mince et riche en LPS se colorent en rose parce que l'alcool, au cours de la coloration, détruit le complexe cristal violet-lugol: les cellules décolorées prennent la couleur rose de la fushine.

L'observation microscopique, après coloration de Gram, donne la forme, la taille et le type de Gram et même la présence ou absence de spores chez certaines souches notamment les lactobacilles.

\section{3-3-2/ Etude des caractères biochimiques des bactéries}

Après avoir isolé une bactérie, il faut procéder à son identification. Une première classification est faite d'après les caractères morphologiques et culturaux puis l'identification des espèces et menée grâce à des milieux d'identification. Certains milieux d'identification sont spécifiques de certains germes, d'autres peuvent servir à de nombreux germes.

La première condition à respecter est d'avoir un germe à l'état "pur", et une culture abondante et suffisante pour ensemencer différents milieux d'identification.

\section{3-3-2-1/ Milieu Kligler-Hajna}

Ce milieu coulé en tube de manière à former un culot et une tranche permet de donner 4 caractères: fermentation du glucose, du lactose, production de gaz et de $\mathrm{H}_{2} \mathrm{~S}$.
Ce milieu à la composition suivante (en $\mathrm{g} / \mathrm{l}$ ) : ( Peptone, 20 ; Nacl, 5 ; Glucose, 1 ; Lactose 10 ; Thiosulfate de sodium, 0,3 ; Citrate ferrique, 0,3 ; Extrait de viande de bœuf, 3 ; Extrait de levure, 3 ; Rouge de phénol, 0,05; Agar, 12).

L'ensemencement se fait au fil droit: une strie sur la tranche et piqûre centrale en profondeur dans le culot. Après 24 heures d'incubation à $37^{\circ} \mathrm{C}$, on obtient les informations suivantes:

* Si la souche est lac (-) et glu (+): il y a d'abord acidification du milieu dans le culot car il y a utilisation du glucose par fermentation, mais cette fermentation est rapidement neutralisé par consommation des peptones dans la pente. En effet, contrairement à la tranche, au niveau du culot il y a une grande quantité de glucose par rapport au nombres de cellules ensemencées, donc durant $24 \mathrm{~h}$ c'est surtout le glucose qui est attaqué.

* Si la souche est lac(+) et glu (+): Il y a acidification par fermentation du glucose prolongée par la suite par celle du lactose au niveau de la tranche. L'acidification se traduit par une coloration jaune du milieu (virage de l'indicateur coloré: rouge de phénol).

* La production de $\mathrm{H}_{2} \mathrm{~S}$ se traduit par noircissement du milieu qui débute à la jonction pente-culot due à la production de sulfate de fer.

* La fermentation des sucres se traduit parfois par production de $\mathrm{CO}_{2}$. On aura soit formation de pochette de gaz dans la gélose ou parfois même soulèvement de la gélose au niveau du culot.

Dans le cas d'identification des vibrion, nous avons utilisé le même milieu de Kligler mais à $3 \%$ de Nacl car les vibrions exigent un milieu de culture salé.

\section{3-3-2-2/ Recherche de l'indole}

Le milieu utilisé est une eau peptonée constituée de peptone pancréatique ou peptone dite bactériologique exempte d'indole.

Le milieu a la composition suivante (en g/l): (peptone exempte d'indole, 10 ; Chlorure de sodium, 5). Le milieu est stérilisé par autoclavage à $120^{\circ} \mathrm{C} / 15 \mathrm{~min}$ est ensemencé et incubé à $37^{\circ} \mathrm{C}$ pendant $24 \mathrm{~h}$. La révélation se fait après l'addition au milieu de $1 \mathrm{ml}$ de réactif de Kovacs dont la composition est la suivante: (Paradimethylaminobenzaldéhyde, $5 \mathrm{~g}$; Alcool amylique,75 ml; $\mathrm{HCl}$ concentré, $25 \mathrm{ml}$ ).

La présence d'indole se manifeste par l'apparition d'une coloration rouge cerise. 
La formation d'indole à partir du tryptophane est un caractère propre à certaines espèces bactériennes, donc utile pour leur identification.

\section{3-3-2-3/ Production de l'uréase}

\section{a/ sur milieu urée de Christensen}

Le milieu à l'urée de Christensen est un milieu solide qui sert à l'identification de certains germes, par la mise en évidence de l'hydrolyse de l'urée.

Formule (en g/l d'eau distillée) : (Peptone, 1 ; Chlorure de sodium, 5 ; Glucose, 1 ; Phosphate monopotassique, 2 ; Urée, 24 ; Rouge de phénol, 0,012 ; Agar, 17,5). Ce milieu est stérilisé à l'autoclave à $120^{\circ} \mathrm{C} / 15 \mathrm{~min}$ et coulé en tubes sous forme de tranche.

La tranche est ensemencée en stries serrées et parallèles avec une culture provenant d'un milieu solide. L'incubation se fait à $37^{\circ} \mathrm{C}$ pendant 1 à 6 jours tout en évitant la dessiccation du milieu.

L'hydrolyse de l'urée se traduit par une alcalinisation du milieu qui prend une teinte rosée, puis rouge, d'abord sur la tranche puis dans le culot (ex. Proteus).

\section{Sur milieu de Stuart (test rapide):}

C'est un milieu liquide, jaune orangé, synthétique, de $\mathrm{pH}$ 6,8 contenant de l'urée, des phosphates disodiques et monopotassiques, l'extrait de levure et le rouge de phénol. Le milieu est stérilisé par filtration.

On incube à $37^{\circ} \mathrm{C}$ une suspension de germes dans 0,5 à 1 $\mathrm{ml}$ de milieu Stuart.

Si le germe est capable d'utiliser l'azote de l'urée pour se multiplier, l'uréase élaborée dégradera l'urée selon la réaction suivante:

$$
\mathrm{H}_{2} \mathrm{O} \quad \mathrm{CO}\left(\mathrm{NH}_{4}\right)_{2} \longrightarrow \mathrm{CO}_{2}+2 \mathrm{NH}_{3}+
$$

Il en résulte une alcalinisation et le milieu deviendra rouge violacé en 3 à 5 heures.

\section{3-3-2-4/ Recherche de la $\beta$-galactosidase (Test O.N.P.G)}

Cette réaction consiste à rechercher directement dans la cellule bactérienne l'existence d'une enzyme permettant l'utilisation du lactose.

Chez les bactéries fermentatives, l'hydrolyse du lactose en glucose et galactose est réalisée par une enzyme intracellulaire : la $\beta$-galactosidase.

Certaines entérobactéries sont dites lactose lent, parce qu'elles possèdent le gène béta-galactose et ne possèdent pas le gène perméase.
En fait les lactoses lents possèdent la possibilité de fermenter le lactose ce qui permet de les rapprocher des germes lactose $(+)$

La mise en évidence de la $\beta$-galactosidase est donc un caractère primordial.

A partir de Kligler, on réalise une suspension dense des bactéries dans $1 \mathrm{ml}$ d'eau distillée. On ajoute ensuite un disque d'ONPG. Si les bactéries possèdent une $\beta$ galactosidase, la solution devient jaune, après un séjour de $20 \min$ à $4 \mathrm{~h} / 37^{\circ} \mathrm{C}$.

\section{3-3-2-5/ Réaction au rouge de méthyle ( $\mathrm{RM})$ et de Vosges-proskauer (V.P)}

On utilise un milieu de culture Clark et Lubs (en g/l ): Peptone, 5; Phosphate bipotassique, 5; Glucose, 5) qu'on inocule par une culture jeune de $18 \mathrm{~h}$. Après incubation de $48 \mathrm{~h}$ à $37^{\circ} \mathrm{C}$, on deux tubes:

* un tube sert à vérifier la production d'acides par addition de Rouge de méthyle $(\mathrm{RM})$ à $0,2 \%$ dans l'alcool à $60^{\circ}$. Si le milieu prend :

- une teinte rouge ( $\mathrm{pH}$ inférieur à 4,2): réaction positive $(\mathrm{RM}+)$

- une teinte jaune ( $\mathrm{pH}$ supérieur à 6,3): réaction négative (RM-)

La réaction de $\mathrm{RM}$ est liée au $\mathrm{pH}$ du milieu. En effet, de nombreuses bactéries produisent des composés organiques acides à partir de l'acide pyruvique.

* dans un deuxième tube on décèle la production d'Acétyl Méthyl-Carbinol ou Acétoïne: Réaction de VosgesProskauer. Ce composé est un produit de dégradation de l'acide pyruvique.

Après 48h d'incubation en Clark et Lubs, $1 \mathrm{ml}$ de milieu et additionné de $0,5 \mathrm{ml}$ d'alpha Naphtol à $6 \%$ dans l'alcool absolu et de $0,5 \mathrm{ml}$ d'une solution aqueuse de $\mathrm{NaOH}$ à $16 \%$. On agite et on attend 15 minutes.

Si la souche étudiée produit de l'acéthylméthyl-carbinol, la présence de ce métabolite se manifeste par l'apparition d'une coloration rouge en surface pouvant diffuser dans le milieu (souche VP+).

\section{3-3-2-6/ Gélose à la phénylalanine désaminase ou gélose APP}

Ce milieu très utile pour la classification des Entérobactéries lac-, permet de rechercher la transformation de la phénylalanine en acide phénylpyruvique.

Ce milieu est composé de (en g/l ): Peptone, 10 ; Phosphate bipotassique, 1 ; Chlorure de sodium, 5 ; Extrait de levure, 3 ; D.L phénylalanine, 2 ; Agar, 12). La stérilisation se fait 
à l'autoclave à $120^{\circ} \mathrm{C}$ pendant 20 minutes puis le milieu est refroidi en position inclinée pour donner une tranche.

Après incubation de 18 à 24 heures à $37^{\circ} \mathrm{C}$, la culture est couverte:

+ Soit avec 5 ou 6 gouttes du réactif suivant : ( Solution de demi-saturation d'alun de fer, $5 \mathrm{ml}$; Sulfate d'ammonium, $2 \mathrm{~g}$; Acide sulfurique à $10 \%, 1 \mathrm{ml}$ ).

+ Soit avec 5 ou 6 gouttes d'une solution au 1/3 de perchlorure de fer.

L'apparition rapide d'une coloration verte franche est caractéristique de la transformation de la phénylalanine en acide phényl-pyruvique.

\section{3-3-2-7/ Milieu citrate de Simmons.}

Ce milieu synthétique permet de savoir si la souche est capable d'utiliser le citrate comme seule source de carbone. Il est composé (en g/l) de: (Sulfate de magnésium, 0,2 ; Citrate de sodium, 2 ; Chlorure de sodium, 5 ; Phosphate d'ammonium, 0,2 ; Phosphate d'ammonium monosodique, 0,8; Bleu de bromothymol, 0,08; Agar, 15). La stérilisation se fait à $121^{\circ} \mathrm{C}$ pendant 15 minutes suivie de refroidissement en position inclinée pour obtenir une pente.

Ce milieu est ensemencé à partir d'une culture prélevée sur milieu gélosé. L'ensemencement se fait en surface par une strie centrale est longitudinale. Après Incubation à l'étuve à $37^{\circ} \mathrm{C}$ pendant $48 \mathrm{~h}$, on observe la couleur et la culture:

* Citrate (+): culture rapide, alcalinisation : milieu bleu

* Citrate (-) : milieu reste vert et sans culture.

\section{3-3-2-8/ Mobilité de la souche}

Nous avons utilisé un milieu de culture dit "gélose mobilité ". Ce milieu a la composition suivante (en g/l) : (peptone, 10 ; NaCl, 5 ; Agar, 5). C'est un milieu mou ( semi solide ) est coulé en tubes et ensemencé par piqûre centrale avec fil droit.

Après incubation de 24 à $48 \mathrm{~h} / 37^{\circ} \mathrm{C}$, lorsque les germes sont mobiles, la culture diffuse de part et d'autre de la piqûre et le milieu devient trouble. Si le germe est peu mobile, seulement quelques bourgeons se développent de part et d'autre de la ligne centrale. S'il est immobile, il cultive uniquement sur le trait de la piqûre.

\section{3-3-2-9/ Mise en évidence des enzymes respiratoires}

\section{a/ Oxydase}

On prélève le germe à partir d'un milieu solide avec öse et on le dépose sur du papier imprégné d'oxalate de dimethylpara-phénylène-diamine, en solution à $1 \%$ dans l'eau distillée stérile.
Le teste se déroule à l'abri de la lumière. Le papier imprégné doit être incolore

Si le germe possède une oxydase, une coloration de la strie en violet survient immédiatement.

Ce test est d'une importance capitale puisque toute la famille des Entérobactériaceae est $\mathrm{OX}^{-}$.

\section{b/ Catalase}

Ce test est pratiqué pour les bactéries bacille ou cocci à Gram +

Le germe est prélevé à partir d'un milieu de culture solide et mis en suspension dans l'eau oxygénée à $10 \%$. La présence de la catalase se traduit immédiatement par dégagement d'oxygène :

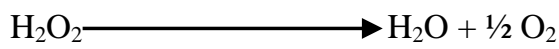

\section{RESULTATS}

La flore présente dans le jus de différentes stations n'est autre que la résultante d'une variété de micro-organismes issus du fruit lui même, de l'atmosphère de stockage, de la chaîne de transformation et de conditionnement, de l'eau et $\mathrm{du}$ personnel. La qualité microbiologique du produit destiné à la consommation est une composante capitale pour garantir une sécurité hygiénique.

\section{6/ Principales bactéries associées aux fruits.}

Les souches fréquemment isolées de différentes étapes de la production de jus d'orange se répartissent en 2 groupes : bacilles à Gram + mais le plus souvent des bacilles à Gram-. Dans ce dernier cas, les bactéries appartiennent à 2 familles les Pseudomonadaceaes et les

Entèrobactériaceaes (tableau 2).

Tableau 1: Origine des souches identifiées

\begin{tabular}{|l|l|}
\hline souches & Origine \\
\hline Pseudomonas sp El 1 & Eau de lavage \\
Pseudomonas sp El 2 & Eau de lavage \\
Pseudomonas sp El 3 & Eau de lavage \\
Klebsiella El 4 & Eau de lavage \\
Pseudomonas sp El 5 & Eau de lavage \\
Enterobacter El 6 & Eau de lavage \\
E. coli El 7 & Eau de lavage \\
Proteus El 8 & Eau de lavage \\
Proteus El 9 & Eau de lavage \\
Serratia El 10 & Eau de lavage \\
Levinia El 11 & Eau de lavage \\
\hline
\end{tabular}




\begin{tabular}{|l|l|}
\hline $\begin{array}{l}\text { Citrobacter El 12 } \\
\text { Baccillus El } 13\end{array}$ & $\begin{array}{l}\text { Eau de lavage } \\
\text { Eau de lavage }\end{array}$ \\
\hline
\end{tabular}

\section{$\boldsymbol{E L}:$ Eau de lavage}

Si les eaux de lavage des fruits renferment une flore très diversifiée et marquées par la présence des Pseudomonas et Klebsiella provenant probablement des eaux d'irrigation ou plutôt des fumiers non traités, au contraire les produits finis ne renferment, après pasteurisation, que les bactéries banales de la familles des Entèrobactèriacaes, sauf un cas où on a trouvé un bacille à endospore cultivant dans une atmosphère normale et dont l'origine peut être attribuée à une contamination par l'air et un traitement inefficace des conduits de la production du jus.

Les souches isolées ont les propriétés biochimiques et physiologiques groupées dans le tableau 2.

Tableau 2: Caractères biochimiques et physiologiques des souches

\begin{tabular}{|c|c|c|c|c|c|c|c|c|c|c|c|c|c|}
\hline \multirow[t]{2}{*}{ test } & \multicolumn{13}{|c|}{ Souches } \\
\hline & El 1 & El 2 & El 3 & $\mathrm{El} 4$ & El 5 & El 6 & El 7 & El 8 & El 9 & El 10 & El 11 & El 12 & El 13 \\
\hline Forme & B & B & B & B & B & B & B & B & B & B & B & B & B \\
\hline Gram & $\mathrm{G}^{-}$ & $\mathrm{G}^{-}$ & $\mathrm{G}^{-}$ & $\mathrm{G}^{-}$ & $\mathrm{G}^{-}$ & $\mathrm{G}^{-}$ & $\mathrm{G}^{-}$ & $\mathrm{G}^{-}$ & $\mathrm{G}^{-}$ & $\mathrm{G}^{-}$ & $\mathrm{G}^{-}$ & $\mathrm{G}^{-}$ & $\mathrm{G}^{+}$ \\
\hline $\mathrm{Ox}$ & + & + & + & + & - & - & - & - & - & - & - & - & \\
\hline Mobilité & ++ & + & $+/-$ & + & ++ & + & + & + & - & ++ & + & - & \\
\hline Glucose & + & + & + & + & + & + & + & + & + & + & + & + & \\
\hline Lactose & + & + & - & - & + & + & - & - & + & - & + & + & \\
\hline $\mathrm{H}_{2} \mathrm{~S}$ & - & - & - & - & - & - & + & + & - & - & + & + & \\
\hline $\mathrm{Gaz}$ & + & + & - & - & + & + & - & + & + & - & - & + & \\
\hline Citrate & & & & & + & - & - & - & & $+/-$ & + & $+/-$ & \\
\hline Indole & & & & & - & + & - & - & & - & + & - & \\
\hline $\mathrm{RM}$ & & & & & - & + & & & & + & + & & \\
\hline VP & & & & & + & - & & & & - & - & - & \\
\hline $\begin{array}{l}\text { Malonate de } \\
\mathrm{Na}\end{array}$ & & & & & & & - & - & & - & & - & \\
\hline Uréase & & & & & - & - & + & + & & - & - & - & \\
\hline ONPG & & & & & + & + & - & - & & - & - & - & \\
\hline King A & + & - & & & & & & & & & & & \\
\hline King B & - & + & & & & & & & & & & & \\
\hline Spore & & & & & & & & & & & & & + \\
\hline
\end{tabular}

\section{DISCUSSION ET CONCLUSION}

Le jus d'orange est riche en eau et en sucres facilement métabolisables par les microorganismes ; c'est un milieu de culture par excellence (Forrest, 2001).

Ces conditions exposent facilement ces produits à la contamination par les champignons et les bactéries pathogènes, une contamination simple ou multiple du Jus peut provenir de nombreuses sources: le sol, l'eau, les engrais (surtout le compost), les travailleurs, le matériel agricole et les conditions de stockage et de transformation.
La flore présente dans le jus n'est autre que le résultante d'une variété de micro-organismes issus du fruit lui même, de l'atmosphère de stockage, parfois de la chaîne de transformation et de conditionnement, de l'eau, de l'air ou même du personnel (Attrassi et Badoc, 2014).

Malgré l'effort entrepris pour réduire la charge des microorganismes dans l'air de l'industries ou pour atténuer la flore liée aux fruits, les quelques cellules de bactéries ou de champignons qui peuvent échapper au contrôle trouvent dans le jus un milieu tout à favorable à leur croissance et à leur reproduction. En effet, le contrôle microbiologique de 
produits finis vise à tuer les microorganismes et inactiver les enzymes responsables de l'altération du produit, mais quelques germes peuvent persister. Ces germes sont en effet considérés loin d'être pathogènes mais sont considérés comme germes nocifs, capables d'altérer le produit à cause de leur métabolisme (D’oust, 1989, 1997).

Les bactéries que nous avons isolées dégradent tout le glucose et se caractérisent par une croissance rapide. Les champignons isolés tolèrent plus l'acidité du milieu (jus d'orange) et peuvent se multiplier sur des substrats plus complexes.

En effet, Salmaoui (1999) avait déjà montré que sur les différentes sources de carbone, les sucres monosaccharides (glucose, fructose et galactose) et le disaccharide (saccharose) sont les plus assimilés par les différents champignons testés (Chapot et Dleuchi, 1964). Le glucose est biologiquement la meilleure source de carbone pour la croissance mycélienne des champignons, ce qui concorde avec le concept général des champignons "tous les champignons possèdent la capacité de convertir le fructose en un dérivé phosphoryle du glucose capable de s'intégrer dans la principale voie de la chaîne respiratoire des enzymes extra cellulaire chez les souches capables de dégrader les polysaccharides" (Attrassi et Rahouti, 2016).

\section{REFERENCES}

[1] ATTRASSI K. et BADOC A., 2014. Moisissures des fruits de deux agrumes. Bull. Soc. Pharm. Bordeaux, 153(1-4), 2534.

[2] ATTRASSI K. et RAHOUTI M., 2016. Effet de composes calciques inorganiques sur le développement in vitro de moisissures isolées d'agrumes après la récolte. Bulletin de la Société Royal des Sciences de Liège, 85 : 253-275.

[3] BEUCHAT L. R. \& RYU J. H., 1997. Produce handling and processing practices. Emerg. Infect. Disc., 3 (4): 459-465

[4] CHAPOT H. \& DLEUCHI .V.L, 1964. Maladies, Troubles et Ravageurs des Agrumes au Maroc, Institut National de la Recherche Agronomique (INRA), Rabat, pp 75-86.

[5] D’OUST J.Y., 1989. Salmonella (eds.) Food borne Bacterial pathogens. Marcel Dekker Inc. Newyork, US.

[6] D’OUST J.Y., 1997. Salmonella species (eds.) Food Microbiology: Fundamentals and frontiers. American society for Microbiology, Washington, D.C.

[7] DOUYLE M.P. \&PADHYE V.V., 1989. Esherichia coli Food borne Bacterial pathogens.(eds.).Mareel Dekker Inc.

[8] DOUYLE M.P. \& CLIVER D.O., 1990. Esherichia coli, D.O. Cliver (ed.) Academic.Press, San Diago, California.

[9] FARBER, J.M., 1989. Food borne pathogenic microorganisms: Characteristics of the organisms and their associated diseases .I. Bacteria. Journal de l'Institut Canadien de sciences et de technologies alimentaire 22 (4): 311-321.
[10] FORREST M.R., 2001. Production et distribution de jus au Canada. Santé Canada. vol. 61, pp1075-1086.

[11] JACKSON E., 2001. Traitement thermique, pasteurisation et autre technologie de transformation, Centre de recherche alimentaire et horticole de l'atlantique, kentiville, nouvelle Ecose $\left(\mathrm{B}_{4} \mathrm{~N}_{1} \mathrm{~J}_{5}\right)$, http://www.cfis.agr.ca/francais/regcode/hrt/juc_frmf.htm

[12] SELMAOUI K., 1999. Etude d'un complexe fongique responsable des pourritures des pommes en conservation. Application de quelques moyens de lutte chimique, thèse de doctorat en Botanique, Université IBN Tofail faculté de science Kenitra, 175p.

[13] TABAGHT L., 1994. Pourritures Noires des fruits d'agrumes, Etiologie de la maladie et possibilité de lutte chimique, thèse de $3^{\text {éme }}$ cycle, Université IBN Zohr faculté de science d'Agadir. 